anales de psicología, 2016, vol. 32, n 2 (mayo), 609-616 http://dx.doi.org/10.6018/analesps.32.2.204941
(C) Copyright 2016: Servicio de Publicaciones de la Universidad de Murcia. Murcia (España) ISSN edición impresa: 0212-9728. ISSN edición web (http://revistas.um.es/analesps): 1695-2294

\title{
Relación entre conducta prosocial, resolución de problemas y consumo de drogas en adolescentes
}

\author{
Olga Hernández-Serrano ${ }^{1 *}$, José P. Espada ${ }^{2}$ y Alejandro Guillén-Riquelme ${ }^{2}$ \\ ${ }^{1}$ Escola Universitària de la Salut i l'Esport. Universitat de Girona (España). \\ ${ }^{2}$ Universidad Miguel Hernández (España).
}

\begin{abstract}
Resumen: Este estudio presenta como objetivos: 1) estudiar el consumo de drogas, la conducta prosocial y la resolución de problemas en función del sexo y la edad, y 2) analizar la asociación de la conducta prosocial y las habilidades de resolución de problemas con el consumo de drogas. Se realizó un estudio descriptivo con un diseño transversal. La muestra se compuso de 567 escolares de Educación Secundaria Obligatoria (48.14\% chicos) con edades entre 14 y 17 años $(M=14.92 ; D T=.90)$. Se hallaron diferencias estadísticamente significativas en el consumo de alcohol y conducta prosocial en función del sexo, así como en el consumo de alcohol y cannabis en función de la edad. Cuando se controló el efecto de la edad y el sexo dentro de los modelos de regresión, la variable que mejor predijo el consumo de alcohol y cannabis fue la conducta prosocial. Los resultados de este estudio resaltan la importancia de promover conductas prosociales como factor de protección del consumo de alcohol y cannabis en la etapa adolescente.

Palabras clave: adolescencia; consumo de drogas; conducta prosocial; resolución de problemas.
\end{abstract}

\section{Antecedentes}

La mayor parte de los estudios recientes sobre el consumo de drogas en la etapa adolescente se centran en los factores de riesgo como las conductas de tipo antisocial (Brindas, Rodríguez, López-Cepero, Rodríguez y Estrada, 2012; Contreras, Molina y Cano, 2012; López y Rodríguez-Arias, 2012; Torregrosa, Inglés, García-Fernández, Gázquez, Díaz y Bermejo, 2012). La evidencia empírica sobre estas conductas de riesgo es más consistente que en el caso de los factores de protección del consumo de drogas como son la conducta prosocial y las estrategias de resolución de problemas (Fang, Barnes-Ceeny y Schinke, 2011; López y Rodríguez-Arias, 2010; Triplett y Payne, 2004). Por ello, el interés de este estudio radica en identificar y valorar en qué medida la conducta prosocial y las estrategias para resolver problemas predicen el consumo de alcohol y otras drogas ilegales en población adolescente. Determinar la relación entre el desarrollo de una conducta voluntaria dirigida a beneficiar a otros, el desarrollo de estrategias de resolución de problemas, y las conductas de consumo de sustancias en adolescentes puede mejorar la eficiencia de las futuras intervenciones preventivas.

* Dirección para correspondencia [Correspondence address]

Dra. Olga Hernández Serrano. Escola Universitària de la Salut i l’Esport (EUSES). Universitat de Girona. Campus de Salt. C/ Francesc Macià, 56. CP.17190 Salt, Girona (España).

E-mail: olga.hernandez@cadscrits.udg.edu
Title: Relationship of the prosocial behaviour, the problem-solving skills and the use of drugs amongst adolescents.

Abstract: The objectives of the present study are the following ones: 1) to study the use of drugs, the prosocial behaviour and the problem-solving skills with respect to age and gender, and 2) to analyze the association of both the prosocial behaviour and the problem-solving skills with the use of drugs. An descriptive cross-sectional study was performed, amongst a sample of 567 students in Spanish Compulsory Secondary Education $(48.14 \%$ males) with an age range from 14 to 17 years $(M=14.92 ; S D=$ .90). Statistically significant differences were found concerning alcohol use and the prosocial behaviour with respect to gender, as well as concerning alcohol and cannabis use with respect to age. When we controlled the effect of age and sex in the regression models, the variable that best predicted alcohol and cannabis use was prosocial behavior. The results from this study enhance the importance of tailored interventions based on the promotion of prosocial behaviours as fundamental protective factor for substances use such as alcohol and cannabis amongst adolescents.

Key words: adolescence; drug use; prosocial behaviour; problem solving.

\section{Importancia del consumo de drogas en adolescentes}

El consumo de drogas es un problema de salud pública que afecta en particular a la población adolescente al presentar una mayor vulnerabilidad (Sussman, Unger y Dent, 2004). En España, un 75\% de estudiantes de 14 a 18 años ha consumido alcohol alguna vez, un $33 \%$ cannabis, y entre $1 \%$ y $3.9 \%$ otras sustancias ilegales como son la cocaína, el éxtasis, los alucinógenos o las anfetaminas. El consumo actual (últimos 30 días) sigue siendo elevado con un $63 \%$ en consumo de alcohol, $17.2 \%$ en cannabis, y entre $0.5 \%$ y $1.5 \%$ en el resto de sustancias. Aunque se ha producido una disminución del consumo a lo largo de los últimos 15 años, el alcohol entre las sustancias legales y el cannabis entre las sustancias ilegales siguen siendo las drogas de mayor prevalencia de consumo en España (Observatorio Español de la Droga y las Toxicomanías [OEDT], 2011). Asimismo, resultan relevantes las diferencias intersexuales en los patrones de consumo, hallándose en los chicos una mayor proporción de consumo de drogas ilegales que en las chicas, incrementándose a medida que aumenta la edad (Miller, Naimi, Brewer y Everett, 2007; OEDT, 2011). En este sentido, diversos autores destacan la importancia de diseñar programas preventivos teniendo en cuenta los diferentes patrones de consumo de sustancias entre hombres y mujeres, reduciendo así el riesgo de mantener una trayectoria de consumo en la edad adulta (Khan, Cleland, Scheidell y Berger, 2014) y las importantes interferencias negativas sobre la salud física y psicológica del adolescente (Brown, 2008; Masten y Faden, 2008). Asimismo, se ha asociado con la participación en conductas de riesgo como por ejemplo absentismo escolar, desvinculación con la escue- 
la, violencia sexual y de pareja, o mayor riesgo de abuso de sustancias (Miller et al., 2007).

\section{Importancia de la conducta prosocial, la resolución de problemas, y el consumo de drogas en adolescen- tes}

La conducta prosocial se asocia con el establecimiento de conductas de carácter voluntario y empáticas dirigidas al beneficio de otros (Eisenberg, Fabes y Spinrad, 2006), tales como ayudar en los deberes o escuchar a otros cuando quieren hablar sobre un problema. Un alto porcentaje de estudiantes españoles de Educación Secundaria (17.35\%) presenta conductas prosociales (Inglés et al., 2008). Asimismo, en la etapa del desarrollo adolescente este tipo de conducta se asocia estrechamente con aspectos académicos tales como el éxito en los estudios (Inglés et al., 2009), la promoción de la competencia social y académica (Eisenberg et al., 2006), la motivación académica (Inglés, Martínez, Valle, GarcíaFernández y Ruíz-Esteban, 2011), las atribuciones académicas internas (Redondo, Inglés y García-Fernández, 2014), y determinadas estrategias y habilidades de estudio, control del tiempo y concentración (Inglés, Martínez-González y GarcíaFernández, 2013). También con aspectos personales y sociales como el autoconcepto (Inglés, Martínez-González, García-Fernández, Torregrosa y Ruiz-Esteban, 2012), la empatía (Mestre, Samper y Frías, 2002; Sánchez-Queija, Oliva y Parra, 2006), la responsabilidad personal y social (Gutíerrez, Escartí y Pascual, 2011), las creencias sobre autoeficacia social (Bandura, Caprara, Barbaranelli, Gerbino y Pastorelli, 2003; Caprara y Steca, 2005) y la mejora del clima social en el aula (Romersi, Martínez-Fernández y Roche, 2011). A su vez, algunos investigadores no encuentran una relación estadísticamente significativa entre la conducta prosocial y el consumo de drogas de los adolescentes (Inglés et al., 2007; La Greca, Prinstein y Fetter, 2001; Martínez-González, RoblesLozano y Trujillo, 2003), por lo que la identifican como un factor protector de las conductas de consumo de drogas (Xue, Zimmerman y Caldwell, 2007). No obstante, las conductas socialmente adaptativas no siempre se han vinculado con la protección. En ocasiones este fenómeno ha sido explicado en base a que estos adolescentes prosociales suelen presentar un mayor número de relaciones interpersonales positivas por lo que podría incrementar la oportunidad de consumir sustancias (Salamó, Gras y Font-Mayolas, 2010; Wild, Flisher, Bhana y Lombard, 2004). En esta misma línea, Gómez-Acosta y Londoño (2013) llevaron a cabo un estudio con una muestra de estudiantes de Educación Secundaria en Bogotá encontrando relación entre el consumo de alcohol y la conducta prosocial cuando este consumo se daba de modo responsable. Así, el bajo nivel de consumo de alcohol predijo en un $98 \%$ la conducta prosocial de los adolescentes. Otros autores ponen de manifiesto la relación entre la recompensa por la implicación prosocial de la comunidad y un mayor consumo de alcohol (López y Rodríguez-Arias, 2010). Además, muchos estudios indican que la conducta prosocial ha- bría que considerarla de modo distinto dependiendo de si es hombre o mujer y del curso académico. Así, la edad y el sexo resultan variables relevantes de forma que este tipo de comportamiento tiende a ser más prevalente entre las mujeres (Calvo, González y Martorell, 2001; Inglés et al., 2008; Sánchez-Queija et al., 2006), y en adolescentes de mayor edad (Eisenberg et al., 2006; Inglés et al., 2008).

Desde el modelo de competencia se defiende una conceptualización positiva de la salud, recayendo el interés en las competencias de los individuos, en lugar de sus déficits. Así, con la llegada de la psicología positiva se identificaron y estudiaron factores protectores del consumo de sustancias como los estilos de afrontamiento centrados en la resolución de problemas. La resolución de problemas es una estrategia de intervención encaminada a ayudar al sujeto a hacer disponibles una variedad de alternativas de respuesta para enfrentarse a una situación problemática y de este modo incrementar la probabilidad de seleccionar la respuesta más adecuada de entre las alternativas posibles (D'Zurilla y Goldfried, 1971). Esta estrategia estaría dentro de las llamadas habilidades para la vida que a su vez se incluyen dentro de los programas de prevención de drogas más efectivos (Botvin y Griffin, 2005; Gómez-Fraguela, Luengo, Romero y Villar, 2003). Según Goldfried y Davison (1981) la finalidad preventiva de la resolución de problemas es mejorar las habilidades de los sujetos para enfrentarse a situaciones problemáticas del día a día. Se considera que es una habilidad fundamental en la etapa adolescente, pues permite tomar decisiones responsables, proporcionando mayor autonomía y ayudando a prevenir problemas. Un estudio meta-analítico realizado en España mostró que entre los componentes más comúnmente utilizados en los programas de prevención del consumo de drogas en adolescentes se halla el entrenamiento en resolución de problemas (Espada et al., 2002). Recientemente, un estudio longitudinal presentó evidencia del impacto del componente de entrenamiento en resolución de problemas sobre variables mediadoras y de resultado en el consumo de drogas a través del desmantelamiento de un programa preventivo del consumo de drogas en adolescentes (Espada, Griffin, Pereira, Orgilés y García-Fernández, 2012; Espada y Méndez, 2003). Pérez de la Barrera (2012) encuentra que los adolescentes con puntuaciones más altas en resolución de problemas fueron los estudiantes no consumidores de marihuana y otras sustancias. En esta misma línea, otros estudios también defienden que un estilo de afrontamiento productivo, como por ejemplo concentrarse en solucionar problemas, se asocia con la protección del consumo de drogas (Gómez-Fraguela, Luengo-Martín, Romero-Triñanes, Villar-Torres y SobralFernández, 2006). Sin embargo, algunos trabajos de investigación no dejan del todo claro que la forma de solucionar problemas por parte de los adolescentes se vincule al no uso de sustancias (Fang et al., 2011; Triplett et al., 2004). En relación a las diferencias demográficas, autores como Garaigordobil y Maganto (2011) analizan la posible existencia de diferencias en el uso de diversas estrategias de resolución de conflictos en función del sexo y la edad. Estos autores en- 
cuentran que las chicas puntúan más alto que los chicos en la utilización de estrategias cooperativas de resolución de conflictos, sin encontrar variaciones a medida que aumenta la edad. Por el contrario, otros trabajos indican que la resolución de problemas se asocia con el desarrollo de estrategias más constructivas a medida que incrementa la edad del adolescente (Owens, Daly y Slee, 2005).

\section{El presente estudio}

A partir de esta contextualización, los objetivos del presente estudio son: 1) estudiar el consumo de drogas, la conducta prosocial y la resolución de problemas en función del sexo y la edad; 2) analizar la asociación de la conducta prosocial y las habilidades de resolución de problemas con el consumo de drogas. A partir de los hallazgos de investigaciones previas, se espera que: 1) el porcentaje de consumidores de drogas, estudiantes con conducta prosocial y estudiantes con habilidades de resolución de problemas sea significativamente diferente en función del sexo y la edad. Así, las chicas presentarán un mayor porcentaje de conductas de tipo prosocial, estrategias de resolución de problemas y menor porcentaje de consumo de drogas ilegales, que los chicos; 2) los adolescentes de mayor edad presentarán un mayor porcentaje de conductas de tipo prosocial, estrategias de resolución de problemas y consumo de drogas; 3) se espera encontrar asociación entre la conducta prosocial, las estrategias para resolver problemas y el consumo de drogas, de modo que la conducta prosocial y las estrategias de resolución de problemas resulten protectoras frente al uso de drogas.

\section{Método}

\section{Participantes}

La muestra se reclutó en cinco centros públicos de Educación Secundaria de áreas rurales y urbanas en la provincia de Alicante. Se llevó a cabo un estudio descriptivo con un diseño transversal durante el curso académico 2011-2012. La muestra estuvo formada por 567 estudiantes (273; 48.14\% varones y 294; $51.85 \%$ mujeres) de Educación Secundaria. Las edades oscilaron entre 14 y 17 años $(M=14.92 ; D T=$ $0.9)$ de $3^{\circ}$ y $4^{\circ}$ de Enseñanza Secundaria Obligatoria (E.S.O). La prueba Chi-cuadrado de homogeneidad de la distribución de frecuencias reveló la ausencia de diferencias estadísticamente significativas entre seis grupos de sexo y curso $\left(\chi^{2}(3)=\right.$ $3.02 ; p>.05)$.

\section{Instrumentos}

Cuestionario de Consumo de Drogas (Espada, Méndez e Hidalgo, 2003). Está compuesto por 13 ítems de respuesta que evalúan variables relacionadas con el consumo alguna vez en la vida (si/no), el consumo alguna vez en el último año (si/no) y la frecuencia de consumo en el último año (alcohol, cannabis, cocaína, éxtasis y alucinógenos). Las cuestiones respecto a la frecuencia del consumo presentan una escala con seis alternativas de respuesta (no consumo, menos de una vez al mes, una vez al mes, una vez a la semana, $2-3$ veces a la semana y diariamente).

Inventario de Habilidades Sociales para Adolescentes (Teenage Inventory of Social Skills, TISS; Inderbitzen y Foster, 1992; adaptación de Inglés, Hidalgo, Méndez e Inderbitzen, 2003). Esta prueba evalúa la competencia social de los adolescentes en las relaciones con sus iguales. Consta de 40 ítems con una escala de 6 puntos $(1=$ no me describe nada; $6=$ me describe totalmente). Está compuesta por dos subescalas:

Conducta prosocial (evalúa comportamientos positivos que generan aceptación por los compañeros) y Conducta antisocial (evalúa comportamientos negativos que generan rechazo por los compañeros), cuyas puntuaciones se obtienen sumando los valores asignados por los sujetos a los 20 ítems que componen cada dimensión. Puntuaciones altas indican una elevada conducta prosocial y antisocial, respectivamente. Las propiedades psicométricas del TISS han sido analizadas en muestras de adolescentes españoles (Inglés, Hidalgo, Méndez e Inderbitzen, 2003) y norteamericanos (Inderbitzen y Foster, 1992), siendo su fiabilidad y validez de constructo satisfactorias. En el presente estudio se utilizó únicamente la escala de Conducta Prosocial.

Cuestionario de Evaluación de Habilidades de Solución de Problemas Sociales, adaptado (SPSI-R; Maydeu y D Zurilla, 1996). Se ha utilizado una adaptación del cuestionario SPSI-R original que permite evaluar la habilidad de solución de problemas en términos de pros y contras y de generación de alternativas a través de 11 ítems con tres alternativas de respuesta (casi nunca, a veces, casi siempre). Puntuaciones altas indican una elevada habilidad para solucionar problemas sociales.

\section{Procedimiento}

La evaluación y la intervención se realizaron en el contexto escolar. El estudio fue aprobado por el Comité de Ética en la Investigación Experimental de la Universidad Miguel Hernández (Elche). Se llevó a cabo una entrevista con el equipo directivo y orientador educativo de los centros participantes para exponer los objetivos y metodología de la investigación, y promover su colaboración. Posteriormente, se informó mediante una circular a los padres solicitando su consentimiento informado por escrito autorizando a sus hijos a participar en el estudio. Licenciados en Psicología llevaron a cabo la administración de las pruebas de forma colectiva y anónima, verificando la cumplimentación independiente de la prueba.

\section{Análisis de datos}

Se llevaron a cabo diversos análisis por sustancias. En el caso de los ítems relacionados con el consumo de cocaína, éxtasis y alucinógenos, el número de consumidores era muy bajo $(\mathrm{n}<5)$, por lo que se descartó la inclusión en el proceso de análisis de las citadas sustancias, al no poder asumirse el 
cumplimiento de supuestos. Posteriormente, se realizaron análisis exploratorios iniciales de las muestras con el fin de controlar el efecto en el resto de análisis. Así, se analizaron las diferencias en las variables de consumo de alcohol y cannabis en función del sexo y la edad (14-17 años), a través de pruebas estadísticas diferentes en función del carácter continuo (t-Student) o categórico (Chi-cuadrado de Pearson) de la variable de consumo estudiada. Atendiendo a las orientaciones de Cohen los tamaños del efecto a través de la prueba $\mathrm{V}$ de Cramer son interpretados del siguiente modo: (1) valores entre 0 y .1 indican que no existe relación; (2) valores entre .1 y .3 indican un efecto pequeño; (3) valores entre .3 y .5 un efecto mediano; (4) valores entre .5 y 1 un efecto grande, y (5) un valor igual a 1 indica una relación perfecta. Para analizar si existían diferencias en las estrategias de resolución de problemas y conducta prosocial en función del sexo se empleó la prueba t-Student y en función de la edad se realizaron análisis de varianza (ANOVA).

Por otro lado, para evaluar el posible efecto de la capacidad de resolución de problemas y la conducta prosocial sobre el consumo (si/no) de alcohol y cannabis (alguna vez en la vida y alguna vez en el último año) se procedió a la construcción de modelos de regresión logística binaria jerárquica, mediante el método "introducir". Se introdujeron en primer lugar las variables demográficas sexo y edad, y en el segundo bloque las variables conducta prosocial y resolución de problemas. Respecto a la frecuencia de consumo de alcohol y cannabis en el último año, teniendo en cuenta que las cuestiones tenían 6 categorías de respuesta, se empleó regresión lineal múltiple.

\section{Resultados}

Consumo de drogas, conducta prosocial y resolución de problemas en función del sexo y la edad

Se analizó la influencia de la edad y el sexo sobre el consumo de alcohol y cannabis, así como sobre la conducta prosocial y las habilidades de resolución de problemas. La Tabla 1 muestra cómo los análisis efectuados revelaron diferencias estadísticamente significativas en las variables de consumo de alcohol en función del sexo y la edad. Las chicas presentaron un mayor porcentaje de consumo de alcohol ocasional $\left(\chi^{2}(\mathrm{gl})\right.$ $=14.98(2) ; p=.00)$ y en el último año $\left(\chi^{2}(\mathrm{gl})=14.10(2) ; p=\right.$ $.00)$ que los chicos, aumentando los porcentajes a medida que aumenta la edad tanto del consumo de alcohol ocasional $\left(\chi_{(\mathrm{gl})}^{2}=24.41(3) ; p=.00\right)$ como en el último año $\left(\chi^{2}(\mathrm{gl})=\right.$ 22.16(3); $p=.00)$. La frecuencia del consumo de alcohol, destacando el consumo una vez a la semana, fue más frecuente entre las chicas que los chicos $\left(\chi^{2}(\mathrm{gl})=27.48(10) ; p=\right.$ $.00)$ aumentando progresivamente con la edad $\left(\chi_{(\mathrm{gl})}^{2}=\right.$
76.38(15); $p=.00)$. Respecto al consumo de cannabis, aunque no se halló diferencias significativas en función del sexo (consumo alguna vez: $\chi^{2}{ }_{(\mathrm{gl})}=.27(2) ; p=.87$; consumo último año: $\left.\chi_{(\mathrm{g})}^{2}=5.81(10) ; p=.83\right)$, los adolescentes de mayor edad presentaron un porcentaje de consumo mayor que los más jóvenes tanto en el consumo de cannabis alguna vez en la vida $\left(\chi^{2}(\mathrm{gl})=32.73(3) ; p=.00\right)$ como en el último año $\left(\chi^{2}(\mathrm{gl})\right.$ $=33.28(3) ; p=.00)$. La frecuencia de consumo de cannabis entre los adolescentes aumenta a medida que incrementa la edad $\left(\chi^{2}(\mathrm{gl})=47.91(15) ; p=.00\right)$. Tanto en el consumo de alcohol como en el consumo de cannabis, todas las diferencias halladas presentaron un tamaño del efecto pequeño.

A continuación, se analizó si había diferencias en la resolución de problemas y conducta prosocial en función del sexo y la edad. En el caso del sexo se observó diferencias estadísticamente significativas en la conducta prosocial $\left(t_{(478)}=\right.$ $5.60 ; p<.00 ; r=.23)$ de las chicas $(M=83.96 ; D T=14.49)$ frente a los chicos $(M=76.78 ; D T=15.98)$, pero no en habilidades de resolución de problemas $\left(t_{(478)}=-.84 ; p=.84\right)$. Asimismo, no se hallaron diferencias en ningún caso en función de la edad (resolución de problemas: $F_{(4,479)}=.96 ; p=$ .97 ; conducta prosocial: $\left.F_{(4,479)}=-.14 ; p=.96\right)$.

\section{Relación entre consumo de drogas, conducta proso- cial y resolución de problemas}

En la Tabla 2 se muestran los resultados de los análisis de regresión logística jerárquica para predecir el consumo de alcohol y cannabis (si/no) alguna vez en la vida y en el último año. Al introducir en un primer momento la variable sexo y edad dentro del modelo de regresión, ambas se mostraron factores relevantes en la predicción del consumo de alcohol de los adolescentes. En la predicción del consumo de cannabis, únicamente la variable demográfica edad se mostró notable. Al controlar sexo y edad, la variable que mejor predijo el consumo de alcohol y cannabis en todos los momentos temporales de medida fue la conducta prosocial. La variabilidad explicada por los modelos según el índice de Nagelkerke fue del 11.1\% y del $8.7 \%$ para el consumo de alcohol alguna vez y el último año respectivamente, y del $7.0 \%$ y del $3.7 \%$ para el consumo de cannabis alguna vez y el último año respectivamente.

En la Tabla 3 se muestran los resultados de los análisis de regresión lineal múltiple para predecir la frecuencia del consumo de alcohol y cannabis en el último año. La conducta prosocial presentó un efecto significativo respecto a la frecuencia del consumo de alcohol y cannabis con un coeficiente de determinación $\mathrm{R}^{2}$ corregida de 0.057 y de 0.052 respectivamente, explicando las variables $5.7 \%$ y el $5.2 \%$ de la varianza. 
Tabla 1. Número (y porcentaje) de sujetos consumidores de alcohol y cannabis clasificados en función del sexo y la edad.

\begin{tabular}{|c|c|c|c|c|c|c|c|c|c|}
\hline & & $\begin{array}{l}\text { Chicos } \\
\text { N }(\%)\end{array}$ & $\begin{array}{l}\text { Chicas } \\
\mathrm{N}(\%)\end{array}$ & V de Cramer & $\begin{array}{l}14 \text { años } \\
\mathrm{N}(\%)\end{array}$ & $\begin{array}{l}15 \text { años } \\
\mathrm{N}(\%)\end{array}$ & $\begin{array}{l}16 \text { años } \\
N(\%)\end{array}$ & $\begin{array}{c}17 \text { años } \\
\mathrm{N}(\%)\end{array}$ & V de Cramer \\
\hline \multirow{13}{*}{ Consumo alcohol } & Alguna vez en la vida & & & \multirow{3}{*}{$.16^{*}$} & & & & & \multirow{3}{*}{$.20 *$} \\
\hline & Sí & $183(67.3)$ & $239(81.3)$ & & $134(64.73)$ & $160(75.11)$ & $97(86.60)$ & $32(91.42)$ & \\
\hline & No & $89(32.7)$ & $55(18.7)$ & & $73(35.26)$ & $53(24.88)$ & $15(13.39)$ & $3(8.57)$ & \\
\hline & Último año & & & \multirow{3}{*}{$.15^{*}$} & & & & & \multirow{3}{*}{$.19 *$} \\
\hline & Sí & $147(54.0)$ & $203(69.0)$ & & 107 (51.69) & 132 (61.97) & $84(75.00)$ & $28(80.00)$ & \\
\hline & No & $125(46.0)$ & $91(31.0)$ & & $100(48.30)$ & $61(38.02)$ & $28(25.00)$ & $7(20.00)$ & \\
\hline & Frecuencia & & & \multirow[t]{7}{*}{$.22 *$} & & & & & \multirow[t]{7}{*}{$.21 *$} \\
\hline & No consumo & $125(46.10)$ & $91(31.10)$ & & $100(48.30)$ & $81(38.20)$ & $28(25.22)$ & $7(20.00)$ & \\
\hline & Menos $1 \mathrm{vez} / \mathrm{mes}$ & $50(18.50)$ & $92(31.40)$ & & $56(27.05)$ & $56(26.41)$ & $25(22.52)$ & $6(17.14)$ & \\
\hline & $1 \mathrm{vez} / \mathrm{mes}$ & $37(13.70)$ & $39(13.30)$ & & $25(12.07)$ & $32(15.09)$ & $18(16.21)$ & $1(2.85)$ & \\
\hline & $1 \mathrm{vez} / \mathrm{semana}$ & $38(14.00)$ & $59(20.10)$ & & $21(10.14)$ & $31(14.62)$ & $31(27.92)$ & $14(40.00)$ & \\
\hline & $2-3$ veces/semana & $15(5.50)$ & $10(3.40)$ & & $2(.96)$ & $7(3.30)$ & $9(8.10)$ & $7(20.00)$ & \\
\hline & Diariamente & $6(2.20)$ & $2(.70)$ & & $3(1.44)$ & $5(2.35)$ & $0(.0)$ & $0(.0)$ & \\
\hline \multirow{13}{*}{ Consumo cannabis } & Alguna vez en la vida & & & \multirow{4}{*}{.01} & & & & & \multirow{4}{*}{$.24 *$} \\
\hline & Sí & $52(19.10)$ & $58(19.70)$ & & $26(12.56)$ & $37(17.37)$ & $29(25.89)$ & $18(51.42)$ & \\
\hline & No & $220(80.90)$ & $236(80.30)$ & & $181(87.43)$ & $176(82.62)$ & $83(74.11)$ & $17(48.57)$ & \\
\hline & Último año & & & & & & & & \\
\hline & Sí & $37(13.60)$ & $40(13.60)$ & \multirow[t]{2}{*}{.00} & $23(11.11)$ & $23(10.80)$ & $15(13.40)$ & $16(45.70)$ & \multirow[t]{2}{*}{$.24 *$} \\
\hline & No & $235(86.40)$ & $254(86.40)$ & & $184(88.89)$ & $190(89.20)$ & $97(86.60)$ & $19(54.30)$ & \\
\hline & Frecuencia & & & \multirow[t]{7}{*}{.10} & & & & & \multirow[t]{7}{*}{$.16^{*}$} \\
\hline & No consumo & $235(86.40)$ & $254(86.40)$ & & $184(88.88)$ & $190(89.20)$ & $97(86.60)$ & $19(54.28)$ & \\
\hline & Menos $1 \mathrm{vez} / \mathrm{mes}$ & $14(5.10)$ & $20(6.80)$ & & $10(4.83)$ & $10(4.69)$ & $8(7.14)$ & $6(17.14)$ & \\
\hline & $1 \mathrm{vez} / \mathrm{mes}$ & $5(1.80)$ & $9(3.10)$ & & $6(2.89)$ & $2(.93)$ & $2(1.78)$ & $4(11.42)$ & \\
\hline & $1 \mathrm{vez} / \mathrm{semana}$ & $11(4.00)$ & $4(1.40)$ & & $4(1.93)$ & $8(3.75)$ & $1(.89)$ & $2(5.71)$ & \\
\hline & $2-3$ veces/semana & $3(1.10)$ & $4(1.40)$ & & $2(.96)$ & $0(.0)$ & $3(2.67)$ & $2(5.71)$ & \\
\hline & Diariamente & $4(1.50)$ & $3(1.00)$ & & $1(.48)$ & $3(1.40)$ & $1(.89)$ & $2(5.71)$ & \\
\hline
\end{tabular}

${ }^{*} p<.05$

Tabla 2. Análisis de regresión logística jerárquica para predecir el consumo de alcohol y cannabis (alguna vez en la vida y alguna vez en el último año).

\begin{tabular}{|c|c|c|c|c|c|c|c|}
\hline & & $\chi^{2}(\mathrm{gl})$ & B & Wald & $p$ & OR & C.I $(95 \%)$ \\
\hline \multirow{4}{*}{ Alcohol alguna vez en la vida } & $1 \mathrm{Edad}$ & $44.17(3)^{*}$ & .61 & 24.66 & .00 & 1.85 & $1.45: 2.36$ \\
\hline & 1 Sexo & & -21.06 & 17.20 & .00 & .00 & $.00: .00$ \\
\hline & 2 Resolución problemas & & .05 & 2.12 & .14 & 1.05 & $.98: 1.14$ \\
\hline & 2 Conducta prosocial & $75.26(5)^{*}$ & .05 & 28.49 & .00 & 1.05 & 1.03:1.07 \\
\hline \multirow{4}{*}{ Alcohol último año } & $1 \mathrm{Edad}$ & $37.57(3) *$ & .48 & 21.34 & .00 & 1.61 & $1.31: 1.98$ \\
\hline & 1 Sexo & & -21.50 & 15.18 & .00 & .00 & $.00: .00$ \\
\hline & 2Resolución problemas & & .05 & 2.15 & .14 & 1.05 & $0.98: 1.12$ \\
\hline & 2 Conducta prosocial & $65.88(5) *$ & .04 & 26.15 & .00 & 1.04 & $1.02: 1.06$ \\
\hline \multirow{4}{*}{ Cannabis alguna vez en la vida } & $1 \mathrm{Edad}$ & $25.33(3)^{*}$ & .56 & 24.41 & .00 & 1.75 & $1.40: 2.18$ \\
\hline & 1 Sexo & & 19.11 & .19 & .90 & .00 & $.00: .00$ \\
\hline & 2Resolución problemas & & .08 & 3.46 & .07 & 1.08 & $.99: 1.18$ \\
\hline & 2 Conducta prosocial & $54.37(5) *$ & .05 & 25.98 & .00 & 1.05 & 1.03:1.07 \\
\hline \multirow{4}{*}{ Cannabis último año } & $1 \mathrm{Edad}$ & $11.55(3)^{*}$ & .42 & 11.46 & .00 & 1.53 & $1.19: 1.96$ \\
\hline & 1 Sexo & & 18.86 & .03 & .98 & .00 & $.00: .00$ \\
\hline & 2Resolución problemas & & .09 & 3.47 & .07 & 1.09 & $.99: 1.21$ \\
\hline & 2 Conducta prosocial & $40.41(5) *$ & .06 & 25.77 & .00 & 1.06 & 1.03:1.08 \\
\hline
\end{tabular}

$* p<.05$

Tabla 3. Análisis de regresión lineal múltiple para predecir la frecuencia del consumo de alcohol y cannabis (último año).

\begin{tabular}{|c|c|c|c|c|c|c|c|}
\hline & & $\mathrm{F}_{(\mathrm{gl})}$ & $\mathrm{B}$ & Beta & $\mathrm{t}$ & $p$ & C.I $(95 \%)$ \\
\hline \multirow{4}{*}{ Frecuencia consumo alcohol } & $1 \mathrm{Edad}$ & $12.73(2)^{*}$ & .25 & .10 & 2.61 & .00 & $.06: .44$ \\
\hline & 1 Sexo & & .79 & .18 & 4.43 & .00 & $.44: 1.14$ \\
\hline & 2Resolución problemas & & .01 & .01 & .34 & .72 & $-.05: .07$ \\
\hline & 2 Conducta prosocial & $9.50(4)^{*}$ & .02 & .14 & 3.45 & .00 & $.01: .04$ \\
\hline \multirow{4}{*}{ Frecuencia consumo cannabis } & $1 \mathrm{Edad}$ & $4.48(2)^{*}$ & .18 & .12 & 2.97 & .00 & $.06: .31$ \\
\hline & 1 Sexo & & .06 & .02 & .53 & .59 & $-.16: .28$ \\
\hline & 2Resolución problemas & & .02 & .05 & 1.28 & .19 & $-.01: .07$ \\
\hline & 2 Conducta prosocial & $8.79(4) *$ & .02 & .21 & 5.07 & .00 & $.01: .03$ \\
\hline
\end{tabular}




\section{Discusión}

El primer objetivo consistió en estudiar el consumo de drogas, la conducta prosocial y la resolución de problemas en función del sexo y la edad. Como era de esperar, la prevalencia de chicas bebedoras fue sustancialmente superior a la de chicos incrementando el consumo de alcohol con la edad tal y como indican los resultados de otras investigaciones (OEDT, 2011), mientras la proporción de consumidores de cannabis no varió significativamente entre chicos y chicas, pero sí en todas las edades. Si bien se observa en estudios que proporcionalmente más chicos que chicas consumen habitualmente cannabis (Llorens, Tomás, Aguilar y Tortajada, 2011; OEDT, 2011; Martínez, Alonso y Montañés, 2010), también se ha encontrado que con la edad la magnitud de consumo de los que se reconocen usuarios es similar en ambos sexos (Bobes, Bascarán, González y Sáiz, 2000; FontMayolas, Gras, Planes, 2006). Por otro lado, el porcentaje de consumidores de cocaína, éxtasis y alucinógenos fue muy bajo, por lo que no se analizaron sus efectos. Este tipo de sustancias son más prevalentes a edades más avanzadas, entre 17-18 años (OEDT, 2011), lo que justificaría el bajo porcentaje de consumidores en este estudio.

Se observaron diferencias de sexo sobre la conducta prosocial. Estas diferencias hipotetizadas coinciden con los resultados de otros estudios donde las chicas presentan niveles más altos de conducta prosocial que los chicos (Calvo et al., 2001; Inglés et al., 2008; Sánchez-Queija et al., 2006). Por ejemplo, algunos autores encuentran que las chicas presentan un mayor número de conductas prosociales asociadas a la implicación familiar, la religión, las habilidades sociales y la creencia en el orden moral, y los chicos conductas prosociales más vinculadas a la comunidad (López y Rodríguez-Arias, 2010). Estas diferencias se han explicado desde distintas vertientes. Por un lado, las normas sociales y el proceso de socialización influyen de forma distinta en ambos sexos. Las chicas aprenden a inhibir, ceder o anteponer en mayor medida las respuestas emocionales, necesidades y deseos que los chicos (Eisenberg et al., 2006). Por otro lado, existe una mayor empatía en las chicas que en los chicos, que a su vez se asocia con la prosocialidad (Garaigordobil y Maganto, 2011; Sánchez-Queija et al., 2006).

En otro orden, y en contra de lo esperado, los datos muestran que no hubo variabilidad entre chicos y chicas para resolver problemas, a diferencia de otros trabajos que indican que las adolescentes presentan puntuaciones más altas en habilidades de comunicación para resolver problemas (Black, 2000; Garaigordobil y Maganto, 2011; Wied, Branje y Meeus, 2007). Asimismo, el porcentaje de estudiantes con estrategias de resolución de problemas fue similar entre los 14 y los 17 años, apuntando este resultado en la misma dirección que otras investigaciones (Garaigordobil y Maganto, 2011) aunque contradiciendo los hallazgos de otros estudios que informan de una mejora con la edad en las estrategias de resolución de conflictos (Laursen y Collins, 1994; Laursen, Finkelstein y Betts, 2001). En concreto, en el estudio de Garai- gordobil y Maganto (2011) el rango de edad utilizado para la muestra de adolescentes se encuentra entre los 12 y 15 años, a diferencia de este estudio que oscila entre los 14 y 17 años. A su vez, en el estudio de Black (2000) se examinan las diferencias de sexo en adolescentes utilizando un método de evaluación observacional (grabaciones en vídeo) lo que podría dar lugar a diferentes resultados. No obstante, la mayor parte de estos trabajos se llevan a cabo fuera del ámbito español (Black, 2000; Wied et al., 2007) y tienen en cuenta diferentes estilos de resolución de conflictos en las relaciones entre adolescentes y padres (Van Doorn, Branje y Meeus, 2011) o entre adolescentes y amigos (Black, 2000). Los resultados sugieren la necesidad de llevar a cabo más estudios que permitan clarificar dichas incongruencias teniendo en cuenta los diversos estilos de resolución de conflictos, las diferentes etapas de la adolescencia, el método de evaluación utilizado y entre quién/quienes va dirigida la resolución de problemas.

El segundo objetivo consistió en analizar la relación entre la conducta prosocial, la resolución de problemas y el consumo de drogas. Una vez controladas las variables sexo y edad dentro de los modelos de regresión, los datos de este trabajo ponen de manifiesto que la variable que mejor predice el consumo de alcohol y cannabis para todos los indicadores temporales (alguna vez en la vida, alguna vez en el último año y frecuencia de consumo en el último año) es la conducta prosocial. Así, la probabilidad de consumir alcohol o cannabis es menor entre los adolescentes con conductas prosociales. Los resultados hallados son consistentes con los informados por otros investigadores que analizaron la relación entre la conducta prosocial y el consumo de drogas (Inglés et al., 2007; La Greca et al., 2001; Martínez et al., 2003; Xue et al., 2007) así como con los obtenidos en diversos trabajos que asocian la asertividad, variable estrechamente ligada a la prosocialidad, y el consumo de drogas (Velázquez, Arellanez y Martínez, 2012). No obstante, la variabilidad explicada por los modelos llevados a cabo en este estudio es escasa lo que sugiere la existencia de otras variables predictivas relevantes que no se han tenido en consideración. Por otro lado, no se halló relación entre las estrategias para resolver problemas y el consumo de alcohol o consumo de cannabis para ninguno de los indicadores temporales. Estos resultados coinciden con lo detectado por Fang et al. (2011) no hallando diferencias en la capacidad para resolver problemas de las adolescentes consumidoras y no consumidoras de drogas. En este sentido, determinados autores indican que algunos adolescentes consumidores de drogas no definen el consumo de sustancias como un problema, sino como una solución a un problema. Es decir, el consumo de drogas es considerado como un modo de afrontar situaciones problemáticas (Triplett et al., 2004), así como de restablecer el equilibrio y la armonía en la persona sin causar una carga para los demás (D’Avanzo, 1997). Así, en el caso del consumo de alcohol y cannabis, la manera de responder y afrontar los problemas parece tener menor peso que la prosocialidad en la protección del consumo. Los comportamientos prosociales desempeñan un papel fundamental en la formación y aceptación de 
relaciones positivas e interpersonales (Inglés, Delgado, García-Fernández, Ruíz-Esteban y Díaz-Herrero, 2010). En este sentido, los hallazgos encontrados en este estudio resaltan la importancia de promover conductas prosociales en la prevención del consumo de sustancias como alcohol y cannabis. Por otro lado, los datos apuntan la necesidad de que futuras investigaciones clarifiquen qué factores determinan la existencia o no existencia de relaciones entre la habilidad para resolver problemas y el no consumo de drogas en la etapa adolescente.

Los resultados obtenidos deben ser interpretados teniendo en cuenta sus limitaciones para futuras investigaciones. En primer lugar, se trata de un estudio transversal por lo que la ausencia de secuencia temporal no permite diferenciar entre causa y efecto. Sería recomendable la utilización de diseños longitudinales para abordar la relación entre las diferentes variables incluidas en este estudio. En segundo lugar, no

\section{Referencias}

Bandura, A., Caprara, G.V., Barbaranelli, C., Gerbino, M., y Pastorelli, C. (2003). Impact of affective self regulatory efficacy on diverse spheres of functioning. Child Development, 74, 1-14. doi: 10.1111/j.15327795.2005.00087.x

Black, K. A. (2000). Gender differences in adolescents' behavior during conflict resolution tasks with best friends. Adolescence, 35(139), 499-512.

Bobes, J., Bascarán, M.T., González, M.P. y Sáiz, P.A. (2000). Epidemiología del uso/abuso de cannabis. Adicciones, 12 (2), 31-40.

Botvin, G. y Griffin, K. (2005). Prevention science, drug abuse prevention, and life skills training: Comments on the state of the science. Journal of Experimental Criminology, 1, 63-78. doi: 10.1007/s11292-004-6462-y

Brindas, C., Rodríguez, L., López-Cepero, J., Rodríguez, F. J. y Estrada, C. (2012). Consumo de drogas y conducta delictiva: análisis diferencial de la heroína y la cocaína en la trayectoria infractora. Revista Iberoamericana de Psicología y Salud, 3, 39-54.

Brown, S., McGue, M., Maggs, J., Schulenberg, J., Hingson, R., Swartzwelder, S., ... Murphy, S. (2008). A developmental perspective on alcohol and youths 16 to 20 years of age. Pediatrics, 121, 290-310. doi: 10.1542/peds.2007-2243D

Calvo, A.J., González, R. y Martorell, C. (2001). Variables relacionadas con la conducta prosocial en la infancia y adolescencia: Personalidad, autoconcepto y género. Infancia y Aprendizaje, 24(1), 95-111. doi: 10.1174/021037001316899947

Caprara, G.V. y Steca, P. (2005). Affective and social self-regulatory efficacy beliefs as determinants of positive thinking and happiness. European Psychologist, 10, 275-286. doi: $10.1027 / 1016-9040.10 .4 .275$

Contreras, L., Molina, V. y Cano, M. C. (2012). Consumo de drogas en adolescentes con conductas infractoras: análisis de variables psicosociales implicadas. Adicciones, 24(1), 31-38.

D'Avanzo, C. E. (1997). Southeast Asians: Asian-Pacific Americans at risk for substance misuse. Substance Use \& Misuse, 32(7-8), 829-848.

D'Zurilla, T. J. y Goldfried, M. R. (1971). Problem solving and behavior modification. Journal of Abnormal Psychology, 78, 107-126.

Eisenberg, N., Fabes, R. A. y Spinrad, T.L. (2006). Prosocial development. En W. Damon y N. Eisenberg (eds.), Handbook of child psychology, vol. 3: Social, emotional and personality development (pp. 646-718). Nueva York: John Wiley \& Sons.

Espada, J. P., Griffin, K. W., Pereira, J. R., Orgilés, M. y García-Fernández, J. M. (2012). Component Analysis of a School-Based Substance Use Prevention Program in Spain: Contributions of Problem Solving and Social Skills Training Content. Prevention Science, 13, 86-95.

Espada, J. P. y Méndez, F. X. (2003). Prevención del abuso de alcobol y drogas de sintesis. Madrid: Ediciones Pirámide. pueden generalizarse estos resultados en niveles educativos distintos a Educación Secundaria, por lo que investigaciones futuras deberían tratar de replicar nuestros hallazgos en otras muestras representativas más grandes y confirmar si existen o no diferencias en estudiantes de otros niveles educativos. Por otro lado, con el objeto de descartar posibles sesgos debidos a la tendencia de los adolescentes a maximizar los comportamientos prosociales en las medidas de autoinforme, sería conveniente combinar con otro tipo de procedimientos de evaluación, como por ejemplo las escalas de valoración por los iguales.

A pesar de estas limitaciones y consideraciones, el presente estudio resalta la importancia de promover conductas prosociales como un factor de protección a tener en cuenta en futuros programas de prevención del consumo de drogas, en concreto de alcohol y cannabis, en población adolescente.

Espada, J. P., Méndez, F. X., Botvin, G. J., Griffin, K. W., Orgilés, M. y Rosa, A. I. (2002). ¿Éxito o fracaso de la prevención del abuso de drogas en el contexto escolar? Un meta-análisis de los programas en España. Psicología Conductual, 10, 581-602.

Espada, J. P., Méndez, F. X. e Hidalgo, M. D. (2003). Batería de Cuestionarios para la Evaluación del Consumo de Drogas en Adolescentes. R.P.I.MU-0781-2003.

Fang, L., Barnes-Ceeney, K. y Schinke, S. P. (2011). Substance use behavior among early-adolescent Asian American Girls: the impact of psychological and family factors. Women Health, 51(7), 623-42. doi: 10.1080/03630242.2011.616575

Font-Mayolas, S., Eugenia, M. y Planes, M. (2006). Análisis del patrón de consumo de cannabis en estudiantes universitarios. Adicciones, 18 (4), 337-344.

Garaigordobil, M. y Maganto, C. (2011). Empatía y resolución de conflictos durante la infancia y la adolescencia. Revista Latinoamericana de Psicología, 43, 255-266.

Goldfried, M. R. y Davison, G. (1981). Técnicas terapéuticas conductistas. Buenos Aires: Editorial Paidós.

Gómez-Acosta, C. A. y Londoño, C. (2013). Modelo predictor del consumo responsable de alcohol y el comportamiento típicamente no violento en adolescentes. Health and Addictions, 13, 23-34.

Gómez-Fraguela, J. A., Luengo-Martín, M. A., Romero-Triñanes, E. y Villar-Torres, P. (2003). El programa construyendo salud: un programa de prevención del abuso de drogas empíricamente fundamentado. Revista Internacional de Ciencias Sociales y Humanidades, 13(1), 165-202.

Gómez-Fraguela, J. A., Luengo-Martín, M. A., Romero-Triñanes, E., VillarTorres, P. y Sobral-Fernández, J. (2006). Estrategias de afrontamiento en el inicio de la adolescencia y su relación con el consumo de drogas y la conducta problemática. International Journal of Clinical and Health Psychology, 6, 581-597.

Gutíerrez, M., Escartí, A. y Pascual, C. (2011). Relaciones entre empatía, conducta prosocial, agresividad, autoeficacia y responsabilidad personal y social de los escolares. Psicothema, 23, 13-19.

Inderbitzen, H., y Foster, S. L. (1992). The Teenage Inventory of Social Skills: Development, Reliability, and Validity. Psychological Assessment, 4, 451-459. doi: 10.1016/S0140-1971(03)00032-0

Inglés, C. J., Benavides, G., Redondo, J., García-Fernández, J. M., RuizEsteban, C., Estévez, C. y Huescar, E. (2009). Conducta prosocial y rendimiento académico en estudiantes españoles de Educación Secundaria Obligatoria. Anales de Psicología, 25(1), 93-101.

Inglés, C. J., Delgado, B., Bautista, R., Torregrosa, M. S., Espada, J. P., García-Fernández, J. M., Hidalgo, M. D., y García-López, L. J. (2007). Factores psicosociales relacionados con el consumo de alcohol y tabaco en 
adolescentes españoles. International Journal of Clinical and Health Psycho$\log y$, 7, 403-420.

Inglés, C. J., Delgado, B., García-Fernández, J. M., Ruiz-Esteban, C. y Díaz-Herrero, A. (2010). Sociometric types and social interaction styles in a sample of Spanish adolescents. Spanish Journal of Psychology, 13(2), 728-738.

Inglés, C. J., Hidalgo, M. D., Méndez, F. X., e Inderbitzen, H. M. (2003). The Teenage Inventory of Social Skills: Reliability and validity of the Spanish translation. Journal of Adolescence, 26(4), 505-510.

Inglés, C. J., Martínez-González, A. E., García-Fernández, J. M. (2013). Conducta prosocial y estrategias de aprendizaje en una muestra de estudiantes españoles de Educación Secundaria Obligatoria. European Journal of Education and Psychology, 6, 33-53.

Inglés, C. J., Martínez-González, A. E., García-Fernández, J. M., Torregrosa, M. S. y Ruíz-Esteban, C. (2012). La conducta prosocial y el autoconcepto de estudiantes españoles de Educación Secundaria Obligatoria. Revista de Psicodidáctica, 17, 135-156. doi: 10.1387/RevPsicodidact.1861

Inglés, C. J., Martínez, A. E., Valle, A., García-Fernández J. M. y RuizEsteban, C. (2011). Conducta prosocial y motivación académica en estudiantes españoles de Educación Secundaria Obligatoria. Universitas Psychologica, 10(2), 557-571.

Inglés, C. J., Martínez-Monteagudo, M. C., Delgado, B., Torregrosa, M. S., Redondo, J., Benavides, G., ... García-López, L. J. (2008). Prevalencia de la conducta agresiva, conducta prosocial y ansiedad social en una muestra de adolescents españoles: un estudio comparativo. Infancia $y$ Aprendizaje, 31 (4), 449-461.

Khan, M. R., Cleland, C. M., Scheidell, J. D. y Berger, A. T. (2014). Gender and racial/ethnic differences in patterns of adolescent alcohol use and associations with adolescent and adult illicit drug use. The American journal of drug and alcohol abuse,40(3), 213-224. doi: 10.3109/00952990.2014.892950

La Greca, A. M., Prinstein, M. J. y Fetter, M. D. (2001). Adolescent peer crowd affiliation: Linkages with health-risk behaviors and close friendships. Journal of Pediatric Psychology, 26(3), 131-143. doi: 10.1093/ipepsy/26.3.131.

Laursen, B. y Collins, W. A. (1994). Interpersonal conflict during adolescence. Psychological Bulletin, 115, 197-209. doi: 10.1037/00332909.115.2.197

Laursen, B., Finkelstein, B. D. y Betts, N. T. (2001). A developmental metaanalysis of peer conflict resolution. Developmental Review, 21(4), 423-449. doi: $10.1006 /$ drev.2000.0531

Llorens, N., Tomás, S., Aguilar, J. y Tortajada, S. (2011). Consumo de cannabis: ¿los factores de riesgo y protección varían según la edad?. Revista Española de Drogodependencias, 36 (4), 427-438.

López, S. y Rodríguez-Arias, J. L. (2010). Factores de riesgo y de protección en el consumo de drogas en adolescentes y diferencias según edad y sexo. Psicothema, 22, 668-673.

López, S. y Rodríguez-Arias, J. L. (2012). Factores de riesgo y de protección en el consumo de drogas y la conducta antisocial en adolescentes y jóvenes españoles. International Journal of Psychological Research, 5, 25-33.

Martínez-González, J. M., Robles-Lozano, L. y Trujillo, H. M. (2003). Diferencias sociodemográficas y protección ante el consumo de drogas legales. International Journal of Clinical and Health Psychology, 3(3), 461-475.

Martínez, M., Alonso, C. y Montañés, J. (2010). Consumo de tabaco, alcohol y cannabis en adolescentes Castellano-Manchegos. Revista Española de Drogodependencias, 35 (1), 78-91.

Masten, A. y Faden, V. (2008). Underage Drinking: A Developmental Framework. Pediatrics, 121, 235-251. doi: 10.1542/peds.2007-2243A.
Maydeu, A. y D'Zurilla, T. J. (1996). A factor-analytic study of the Social Problem-Solving Inventory: An integration of theory and data. Cognitive Therapy and Research, 20(2), 115-133. doi: 10.1007/BF02228030

Mestre, M. V., Samper, P. y Frías, M. D. (2002). Procesos cognitivos y emocionales predictores de la conducta prosocial y agresiva: La empatía como factor modulador. Psicothema, 14, 227-232.

Miller, J., Naimi, T., Brewer, R. y Everett, S. (2007). Binge Drinking and Associated Health Risk Behaviors Among High School Students. Pediatrics, 119, 76-85._doi: 10.1542/peds.2006-1517

Salamó, A., Gras, M. E. y Font-Mayoles, S. (2010). Patrones de consumo de alcohol en la adolescencia. Psicothema, 22, 189-195.

Sánchez-Queija, I., Oliva, A. y Parra, A. (2006). Empatía y conducta prosocial durante la adolescencia. Revista de Psicología Social, 21, 259-271. doi: 10.1174/021347406778538230.

Sussman, S., Unger, J. B. y Dent, C. W. (2004). Peer group selfidentification among alternative high school youth: A predictor of their psychosocial functioning five years later. International Journal of Clinical and Health Psychology, 4, 9-25.

Observatorio Español de la Droga y las Toxicomanías (2011). Informe 2011. Situación y tendencias de los problemas de drogas en España. Madrid: Ministerio de Sanidad, Política Social e Igualdad.

Owens, L., Daly, A. y Slee, P. (2005). Sex and age differences in victimization and conflict resolution among adolescents in a South Australian school. Aggressive Behavior, 31(1), 1-12. doi: 10.1002/ab.20045.

Pérez de la Barrera, C. P. (2012). Habilidades para la vida y consumo de drogas en adolescentes escolarizados mexicanos. Adicciones, 24(2), 153 160.

Redondo, J., Inglés, C. J. y García-Fernández, J. M. (2014). Conducta prosocial y autoatribuciones académicas en Educación Secundaria Obligatoria. Anales de psicología, 30(2), 482-489. doi: http://dx.doi.org/10.6018/analesps.30.2.148331

Romersi, S., Martínez-Fernández, J. R. y Roche, R. (2011). Efectos del Programa Mínimo de Incremento Prosocial en una muestra de estudiantes de educación secundaria. Anales de Psicología, 27(1), 135-146.

Torregrosa, M. S., Ingles, C. J., García-Fernández, J. M., Gázquez, J. J., Díaz, A. y Bermejo, R. M. (2012). Conducta agresiva entre iguales y rendimiento académico en adolescentes españoles. Psicología Conductual, 20, 263-280.

Triplett, R. y Payne, B. (2004). Problem solving as reinforcement in adolescent drug use: Implications for theory and policy. Journal of Criminal Jus tice, 32(6), 617-630

Velázquez, M., Arellanez, J. L. y Martínez, A. L. (2012). Asertividad y consumo de drogas en estudiantes mexicanos. Acta Colombiana de Psicología, 15(1), 131-141.

Van Doorn, M. D., Branje, S. J. y Meeus, W. H. (2011). Developmental changes in conflict resolution styles in parent-adolescent relationships: A four-wave longitudinal study. Journal of Youth and Adolescence, 40(1), 97-107. doi: 10.1007/s10964-010-9516-7.

Wied, M., Branje, S. J. y Meeus, W. H. (2007). Empathy and conflict resolution in friendship relations among adolescents. Aggressive Behavior, 33(1), 48-55. doi: 10.1002/ab.20166.

Wild, L.G., Flisher, A.J., Bhana, A. y Lombard, C. (2004). Associations among adolescent risk behaviours and self-esteem in six domains. Jour nal of Child Psychology and Psychiatry, 45(8), 1454-1467. doi: 10.1111/j.1469-7610.2004.00330.x.

Xue, Y., Zimmerman, M. A. y Caldwell, C. H. (2007). Neighborhood residence and cigarette smoking among urban youths: the protective role of prosocial activities. American Journal of Public Health, 97(10), 1865 1872. doi: 10.2105/AJPH.2005.081307 